\title{
Monotonic ratios of functions
}

\author{
G.J.O. Jameson
}

Problem: Show that if $p>1$, then $\sinh p x / \sinh x$ increases with $x$ for $x>0$.

The most obvious approach is to try to show that the derivative is non-negative. This can, in fact, be achieved without too much difficulty, using the special properties of the functions sinh and cosh. However, one is left with the feeling that this might be a special case of something much more general. Does a similar statement apply to $f(p x) / f(x)$ for a wide range of functions $f$ ? We will show that this is indeed the case whenever $f$ is a polynomial, or a power series, with non-negative coefficients. In fact, we will establish a more general result applying to suitable ratios $g(x) / f(x)$.

We use the term "increasing" in the wide sense: if $x_{1}<x_{2}$, then $f\left(x_{1}\right) \leq f\left(x_{2}\right)$ (not excluding the case where $f(x)$ is constant). Also, to avoid tedious repetition, we will say, for example, that $f(x)$ "decreases with $x$ " to mean that it is a decreasing function of $x$ (and similarly with $n$ instead of $x$ ). Our result is as follows.

Theorem: (i) Suppose that $f(x)=\sum_{r=0}^{n} a_{r} x^{r}$ and $g(x)=\sum_{r=0}^{n} c_{r} a_{r} x^{r}$, where $a_{r} \geq 0$ and $c_{r}>0$ for each $r$, with some $a_{r_{0}}>0$. If $c_{r}$ decreases with $r$, then $g(x) / f(x)$ decreases with $x$ for $x>0$. If $c_{r}$ increases, then $g(x) / f(x)$ increases.

(ii) Now suppose that $f(x)=\sum_{n=0}^{\infty} a_{n} x^{n}$ and $g(x)=\sum_{n=0}^{\infty} c_{n} a_{n} x^{n}$ for $|x|<R$, where $a_{n} \geq 0$ and $c_{n}>0$ for each $n$, with some $a_{n_{0}}>0$. If $c_{n}$ decreases with $n$, then $g(x) / f(x)$ decreases with $x$ on $(0, R)$. If $c_{n}$ increases, then $g(x) / f(x)$ increases.

Of course, (ii) follows from (i) simply by considering limits.

What happens if one tries to prove the theorem by showing that the derivative is non-negative? This is equivalent to showing that $f(x) g^{\prime}(x)-f^{\prime}(x) g(x) \geq 0$, perhaps by showing that all the coefficients in this expression are non-negative. It turns out that this approach just leads to unpleasantly complicated expressions, with no transparent route to the conclusion. To test this assertion, the reader could try writing out the case $n=3$.

Our method will not use differentiation at all. Instead, we will use Abel summation, which is the following way to rewrite a sum of products. Given $a_{r}, b_{r}$ for $0 \leq r \leq n$, write $A_{r}=a_{0}+a_{1}+\cdots+a_{r}$. Then $a_{0}=A_{0}$ and $a_{r}=A_{r}-A_{r-1}$ for $r \geq 1$, so

$$
\sum_{r=0}^{n} a_{r} b_{r}=A_{0} b_{0}+\left(A_{1}-A_{0}\right) b_{1}+\cdots+\left(A_{n}-A_{n-1}\right) b_{n}
$$




$$
=A_{0}\left(b_{0}-b_{1}\right)+A_{1}\left(b_{1}-b_{2}\right)+\cdots+A_{n-1}\left(b_{n-1}-b_{n}\right)+A_{n} b_{n} .
$$

The other ingredient of our proof is the following obvious fact: if $f(x)$ and $g(x)$ are positive, then $f(x) / g(x)$ is increasing if and only if $g(x) / f(x)$ is decreasing. We will apply this repeatedly, in a switchback ride of successive inversions.

Proof of the Theorem: As already mentioned, we only need to prove (i). Also, it is enough to prove the statement for decreasing $c_{r}$. The statement for increasing $c_{r}$ then follows, by considering $f(x) / g(x)$ and noting that $a_{r}=c_{r}^{-1}\left(c_{r} a_{r}\right)$. Further, if $r_{0}>0$, then division top and bottom by $x^{r_{0}}$ replaces $f(x)$ by a polynomial with non-zero constant term, so it is enough to consider the case where $a_{0}>0$.

Write $f_{k}(x)=\sum_{r=0}^{k} a_{r} x^{r}$ (so $\left.f_{n}(x)=f(x)\right)$. By Abel summation,

$$
g(x)=\sum_{k=0}^{n-1}\left(c_{k}-c_{k+1}\right) f_{k}(x)+c_{n} f(x) .
$$

Since $c_{k}-c_{k+1} \geq 0$, the required statement follows if we can show that for each $k<n$, the ratio $f_{k}(x) / f(x)$ decreases with $x$. Consider the reciprocal:

$$
\frac{f(x)}{f_{k}(x)}=1+\sum_{s=k+1}^{n} \frac{a_{s} x^{s}}{f_{k}(x)} .
$$

Inverting again, we have for $s>k$,

$$
\frac{f_{k}(x)}{x^{s}}=\sum_{r=0}^{k} a_{r} x^{r-s} .
$$

Here $r-s<0$, so $x^{r-s}$ decreases with $x$. Hence $f_{k}(x) / x^{s}$ decreases, so so $x^{s} / f_{k}(x)$ increases. Therefore $f(x) / f_{k}(x)$ increases, so $f_{k}(x) / f(x)$ decreases, as required.

Note. For a minor generalisation, replace the terms $x^{r}$ by positive functions $u_{r}(x)$ satisfying the condition that $u_{r}(x) / u_{r+1}(x)$ decreases with $x$ on $(0, \infty)$. The proof is the same, with $x^{r-s}$ replaced by $u_{r}(x) / u_{s}(x)$.

An immediate deduction is the result we stated first, slightly enhanced:

Corollary: Let $f(x)=\sum_{r=0}^{n} a_{r} x^{r}$, where $a_{r} \geq 0$ for all $r$, with some $a_{r_{0}}>0$. If $p>q>0$, then $f(p x) / f(q x)$ increases with $x$ on $(0, \infty)$. Similarly for infinite series $f(x)=$ $\sum_{n=0}^{\infty} a_{n} x^{n}$ within the interval where $f(p x)$ converges.

Proof: Apply the Theorem with $a_{n}$ replaced by $a_{n} q^{n}$ and $c_{n}=p^{n} / q^{n}$.

In particular, we recover our original example $\sinh p x / \sinh x$, together with (for example) $\cosh p x / \cosh x$. We record a number of other particular cases. 
Example 1: Applied to the infinite geometric series $\sum_{n=0}^{\infty} x^{n}=1 /(1-x)($ for $|x|<1)$, the Corollary says that if $p>q>0$, then $(1-q x) /(1-p x)$ is increasing for $0<x<\frac{1}{p}$. However, this is obvious: the expression equates to $q / p+(p-q) /(1-p x)$. But for the polynomial $f_{n}(x)=1+x+\cdots+x^{n-1}$, the statement is that $f_{n}(p x) / f_{n}(q x)$ increases for all $x>0$, and this is not at all trivial. Again, direct differentiation does not provide an easy proof, and the identity $f_{n}(x)=\left(1-x^{n}\right) /(1-x)$ gives (for $x$ not equal to $\frac{1}{p}$ or $\frac{1}{q}$ )

$$
\frac{f_{n}(p x)}{f_{n}(q x)}=\frac{1-q x}{1-p x} \frac{1-p^{n} x^{n}}{1-q^{n} x^{n}}:
$$

for $x<\frac{1}{p}$, the first factor is increasing (as just seen), while the second factor is decreasing.

Example 2: To simplify notation, write $L(x)=-\log (1-x)$, so that $L(x)=\sum_{n=1}^{\infty} \frac{x^{n}}{n}$ for $|x|<1$. So if $0<p<1$, then $L(p x) / L(x)$ decreases on $(0,1)$. Of course, it follows with no further work that the derivative is non-positive: we can reason this way round just as well as conversely! Written out, this equates to the following inequality:

$$
p(1-x) L(x) \leq(1-p x) L(p x) .
$$

A direct proof of this is possible, but it entails careful comparison of the series expressions for both sides. Also, substituting $x=1 / y$, we deduce that

$$
\frac{\log y-\log (y-p)}{\log y-\log (y-1)}
$$

increases with $y$ for $y>1$.

Example 3: The "dilogarithm function" $\operatorname{Li}_{2}(x)$ is defined for $|x| \leq 1$ by $\operatorname{Li}_{2}(x)=$ $\sum_{n=1}^{\infty} x^{n} / n^{2}$. So our Theorem, with $c_{n}=\frac{1}{n}$, shows that $\operatorname{Li}_{2}(x) / L(x)$ decreases on $[0,1)$. Similarly, for example, $x \cosh x / \sinh x$ increases with $x$.

Some other expressions can be reduced to our type by substitutions. We give two examples.

Example 4: Let $f(x)=\left(x^{p}-x^{-p}\right) /\left(x-x^{-1}\right)$ for $x>1$. The substitution $x=e^{t}$ transforms $f(x)$ to $\sinh p t / \sinh t$, and $x$ increases when $t$ increases, so if $p>1$, then $f(x)$ increases for $x>1$.

Example 5: Let

$$
f(x)=\frac{(x-1)\left(x^{p}+1\right)}{x^{p+1}-1}
$$

for $x>1$. (Note: if $p=1$, then $f(x)$ has the constant value 1.) Substitute $x=e^{2 t}$ : then $f(x)=g(t)$, where

$$
g(t)=\frac{\left(e^{2 t}-1\right)\left(e^{2 p t+1}\right)}{e^{2(p+1) t}-1}=\frac{2 \sinh t \cosh p t}{\sinh (p+1) t}=1-\frac{\sinh (p-1) t}{\sinh (p+1) t},
$$


since $2 \sinh t \cosh p t=\sinh (p+1) t-\sinh (p-1) t$. So if $p \geq 1$, then $g(t)$ increases with $t$, hence $f(x)$ increases with $x$.

Further thoughts about $f(p x) / f(x)$. Let us say that a function $f$ has property $(A)$ if it is positive and for all $p>1$, the ratio $f(p x) / f(x)$ increases with $x$ on the positive interval within its domain of definition. The Corollary says that polynomials and power series with non-negative coefficients have property (A). Are there lots more functions with the property?

A rather trivial answer is that $f(x)=x^{r}$ has the property for any $r$ (positive or negative), since then $f(p x) / f(x)$ has the constant value $p^{r}$. Numerous further examples are now generated by the following observations. First, if $f(x)$ and $g(x)$ have property $(\mathrm{A})$, then so does $f(x) g(x)$. Second, if $f(x)$ has property (A), then so do the functions $f(x)^{r}$ and $f\left(x^{r}\right)$ for all $r>0$. So the following functions all have property (A):

$$
x+\frac{1}{x}, \quad\left(x+\frac{1}{x}\right) \sinh x, \quad(\sinh x)^{1 / 2}, \quad \sinh x^{1 / 2} .
$$

Finally, let us compare property (A) with the class of convex functions. Recall that a differentiable function $f$ is convex if its derivative $f^{\prime}$ is increasing. Hence $x^{r}$ is convex for $r \geq 1$ and $r \leq 0$, and concave for $0 \leq r \leq 1$. So the functions described in the Corollary are certainly convex. But there is no close match. The non-convex functions $x^{r}$, for $0<r<1$, have property (A). Meanwhile, the convex function $e^{-x}$ does not have property (A), since $e^{-p x} / e^{-x}=e^{(1-p) x}$, which is decreasing. Another such example, easily verified, is $1 /(x+1)$.

Acknowledgment: The author is grateful to the referee for a number of useful suggestions.

Dept. of Mathematics and Statistics, Lancaster University, Lancaster LA1 4YF, UK e-mail: g.jameson@lancaster.ac.uk 